\title{
Wealthy and Healthy? New evidence on the relationship between wealth and HIV vulnerability in Tanzania
}

\section{Danya Long}

\section{Kevin Deane}

\begin{abstract}
Using AIS/DHS data for Tanzania in 2003-4, 2007-8 and 2011-12 and borrowing from the methodology used in Parkhurst (2010) we analyse the changing relationship between wealth and HIV prevalence in Tanzania. Findings are tabulated, graphed and discussed.

We find the relationship is multifaceted and dynamic: women are disproportionately affected in all wealth quintiles and experience a stronger 'wealth effect'; some groups experience an increase in prevalence even as population prevalence declines. Relative wealth and poverty are associated with increased prevalence, suggesting that structural drivers create a variety of risk situations - as well as protective factors - affecting different groups.
\end{abstract}

We also consider data on testing refusals: wealthier men were consistently more likely to decline testing. Continuing to unpack this complex and shifting relationship is necessary in order to fully understand the structural drivers of HIV transmission and access of testing sevices, enabling the formulation of appropriate policy responses.

\section{Introduction}

Although there was a general awareness in the early years of the HIVIAIDS epidemic that those of higher socio-economic status, such as school teachers, had high rates of infection (Hamoudi and Birdsall 2004), HIVIAIDS has long been characterised as a disease of poverty, with explanations for and responses to the epidemic framed within a poverty narrative (World Bank 1999; Hope 2001; Whiteside 2002; Masanjala 2007). The evidence, however, does not necessarily support this narrative, with early acknowledgments that the wealthy were impacted (Chao et al. 1994) supplemented with later findings from nationally representative surveys that emphasise the complexity of the relationships between wealth and prevalence (Wojcicki 2005; Mishra et al. 2007; Fortson 2008), and, in some cases, suggest that it is the wealthiest that have the highest prevalence rates (Msisha et al. 2008; Parkhurst 2010). These recent studies typically use data from representative demographic surveys, an improvement on previous methodological approaches that focused on gathering data at antenatal clinics (Bennel 2004), and in general report a range of different correlations between relative wealth and HIV prevalence in a number of countries in sub"aharan Africa. The relationship in some cases is seen to be positively monotonic, other times 'U- shaped' with both the wealthiest and poorest segments of the population experiencing higher rates of HIV prevalence. For example, Parkhurst (2010) finds that HIV prevalence increases across wealth quintiles in a number of countries for men and women, though this correlation differs by both GDP per capita and the HIV prevalence rate of the population as a whole. Mishra et al (2007) find that even when controlling for likely cofactors (for example urban vs. rural dwelling) the 'wealth effect' remains significant. The relationship between wealth and HIV is, statistically, experienced differently by gender; typically relative wealth seems to act more sharply as a 'risk factor' for women than for men. Wojcicki (2005) in a review of 36 studies that specifically focus on the relationship between 
socioeconomic status and HIV for women, reported that 'fifteen found no association between SES and HIV infection, twelve found an association between high SES and HIV infection, eight found an association between low SES and infection, and the results from one were mixed' (Wojcicki 2005) again emphasising that relative wealth is often an important factor, though it was noted that the socioeconomic status of male partners is also influential in shaping patterns of infection (Wojcicki 2005). Whilst the evidence is heterogeneous in that there is no one universal correlation (Parkhurst 2010), contrary to the poverty narrative, 'HIVIAID" does not disproportionally affect the poorer in sub-Saharan Africa' (Mishra et al. 2007).

There are, however, limitations with the data used for these analyses, as they are all based on panel or survey data, and can only give snapshots at a specific point in time, as opposed to longitudinal surveys which track the same population over a sustained period of time and hence can capture changes in incidence rates for different wealth groups. Longitudinal studies are therefore better placed to shed light on how behaviour changes over time, and whether the assumptions noted above are borne out. However, as noted in Johnston (2013), sadly these studies are few and far between, in part inhibited by the cost of repeatedly surveying the same population over a number of years, with the results from the only three available studies portraying a mixed picture (Johnston 2013). Johnston (ibid) notes that whilst one study reports declining incidence for wealthier men but not women (Lopman et al. 2007), two other studies conducted in South Africa over a similar time period reported different results (Hargreaves et al. 2002; Barnighausen et al. 2007). However, neither of the studies conducted in South Africa found a statistical correlation between increasing incidence and poverty, suggesting that this evidence is broadly supportive of the conclusion that Mishra et al (2007) came to with their analysis conducted using prevalence data. Whichever method is used, it seems that the evidence to-date consistently contradicts the poverty-HIV narrative.

However, despite widespread acknowledgment of these findings (Fenton 2004; Shelton et al. 2005; Gillespie et al. 2007), the response to the epidemic remains firmly rooted in a poverty narrative, best illustrated by Fenton's conclusion that despite the data, 'reducing poverty will be at the core of a long-term, sustainable solution to HIVIAIDS (Fenton, 2004, p1187). This is echoed across the literature, with a range of other studies emphasising the role of poverty (Booysen Fle and Summerton 2002; Stillwaggon 2002; Freedman and Poku 2005; Kalichman et al. 2006; Dodoo et al. 2007; Kalipeni and Ghosh 2007; Lopman et al. 2007; Weiser et al. 2007; Chaturaka and Senaka 2010; Mufune 2014). Whilst UNAID“ continues to emphasise the need to 'know your epidemic' and for interventions to be evidence-based (Wilson and Halperin 2008), this advice is not currently being heeded, and hence the poverty narrative has taken on a paradigmatic quality.

Several factors underpin this lack of consideration of the role of relative wealth, and the continued focus on poverty. First, it was assumed that the wealthy would be the first to change their behaviours in response to the epidemic (Bujra 2006), a view couched in rational behavioural terms; the wealthiest will be more likely to respond to educational campaigns, and that they can afford condoms and/or treatment and therefore will do so. However, evidence from recent studies show that risk behaviours are in some cases still correlated positively with wealth (Kongnyuy et al. 2006; Awusabo-Asare and Annim 2008), suggesting that anticipated changes in behaviour have not always materialised. 
A second factor is the observation that wealth is often correlated with negative health outcomes simply because the wealthy live longer (Beegle and de Walque 2009). This issue has been prominent in discussions of the correlations between HIV and wealth, with general agreement that as the wealthy have greater access to ARV treatment and live longer, prevalence rates for the wealthy are biased upwards and thus this is a statistical anomaly that can be ignored. There is certainly a strong degree of truth to this assumption, as demographic evidence across the continent reports longer life expectancies for the wealthy. However, the issue of biases in the data related to post-infection impacts on household income and wealth are often not addressed, despite evidence suggesting that postinfection morbidity is associated with a reduction in household income. For example, Bachmann and Booysen found that income and expenditure was lower in households which had an HIV infected member than those that did not (Bachmann and Booysen 2003), whilst a study on a tea plantation in Kenya found that HIV-infected workers earned significantly lower incomes than other workers in the two years before retirement or death (Fox et al. 2004). Studies on the impact of HIVIAIDS related mortality on households (death rather than illness), emphasise that although household's expenditure/income often recover from suffering an HIVIAIDS related death after a period of around 5 years (Seeley et al. 2010), by inference this suggests that there is a negative morbidity impact to recover from. Although the evidence base for the impact of HIVIAIDS related morbidity is small and in urgent need of additional research, the limited evidence highlights that households do experience reductions in income post-infection, suggesting that this will bias prevalence data for poorer wealth quintiles.

Third, there is evidence to suggest that positive correlations between wealth and HIV status disappear when samples are divided into rural and urban samples, reflecting that fact that the majority of the wealthy live in urban areas (Beegle and de Walque 2009). An analysis of DHS data available for sub-Saharan countries, using a relative measure of wealth, found that in rural areas, poverty was not associated with higher HIV prevalence, but in urban areas the urban poor are disproportionately affected (Magadi 2013). These findings further muddy the picture, illustrating that different forms of poverty may or may not be related to enhanced HIV risk, and that the interaction between poverty and other contextual and structural factors are also important to account for.

A final observation that is often repeated in the literature links HIV and poverty because Africa is both the poorest continent and home to the majority of those living with HIVIAIDS (Mbirimtengerenji 2007), an analysis that focuses on context, rather than patterns of behaviour and outcomes, reflecting the fertile terrain that Stillwaggon (2002) refers to. However, an alternative view would suggest that Africa is home to the majority of those living with HIV because this was where the epidemic originated (Iliffe 2006).

Whilst there are examples of excellent studies that address the role of wealth in the HIV epidemic (Bujra 2006), there are a number of compelling reasons for revisiting this issue. Prominent amongst them is the availability of new data which enables us to update previous work (Parkhurst 2010) to assess to what extent the optimism around behavioural change and declining prevalence rates for the wealthiest has played out. Secondly, there are few, if any, policies that incorporate the wealthy (or 'wealthier') within the target population, with recent economic interventions such as microfinance and cash transfers that are becoming increasingly popular in HIV prevention efforts that seek to address 'structural' drivers (Kim and Watts 2005; Baird et al. 2012), targeted primarily at 
poor women and girls. The advent of treatment as prevention (Cohen et al. 2011; Thigpen et al. 2012), and the global focus on expanding access to $A ' V$ 's for those infected (UNAIDS 2013), to some extent at the expense of prevention efforts, are also more recent themes that require attention, and especially concerning the assumptions around enhanced access of these services by the wealthy (Obermeyer et al. 2013). This has not been addressed in great detail in the literature to date, yet is a crucial component in the overall debate, especially in relation to the question of whether the prevalence data contains biases. Finally, a very limited number of studies have engaged with this topic previously. Bujra (2006) provides a critical analysis of mainstream economic approaches to transmission, and locates the high rates of HIV seen in wealthy women in Tanzania within the context of processes of class formation and the reinforcement of class identity (Bujra 2006). Beyond this, convincing explanations are scarce, especially within the economics discipline, which continues to frame transmission within rational terms (Philipson and Posner 1995; Oster 2005; Oster 2007; Oster 2012), emphasising the need for a renewed focus.

The rest of this article is structured as follows. Firstly, we provide a brief introduction to the history of the epidemic in Tanzania, and then present an updated statistical analysis using recent data from Tanzania. We then critically assess competing explanations for the analysis, before discussing further implications of our results in relation to the current policy agenda and the structural drivers literature (Gupta et al. 2008; Auerbach et al. 2011). It is important to note at this point that we acknowledge poverty will be an important factor in some settings. However, our assertion is that the framing of HIV as a disease of poverty is at best inaccurate, at worst something that diverts attention from the role of wealth, and thus only serves to dilute international efforts to combat the spread and impact of the virus. A renewed focus on the roles of, and interactions between, both wealth and poverty, and how these produce different health outcomes across the population (see O'Laughlin, B, this issue), is required.

\section{The Tanzanian epidemic: New evidence on the relationship between wealth and HIV}

The first cases of AIDS in Tanzania were recorded in Kagera region in 1983 (Ministry of Health 2003; lliffe 2006), though it is likely that the virus entered the Uganda- Tanzania border region in the mid to late 1970's(Iliffe 2006). By 1986, just a few years later, the virus had penetrated all mainland regions (Iliffe 2006) ${ }^{1}$. Whilst is it difficult to build up an accurate picture of prevalence and trends during the 1990's, as prevalence rates were estimates based on measurements taken primarily at Antenatal Clinics, there is some evidence to suggest that prevalence peaked in Tanzania in 2001 (Asamoah-Odei et al. 2004). More recent data, based on the Tanzania HIVIAIDS Indicator surveys of 2003-04, 2007-08 and 2011-12 (discussed in more detail below), show a national prevalence rate of $7 \%$ in 2003 04, that has declined to $5.1 \%$ in 2011-12 (TACAIDS 2005; TACAIDS et al. 2013). At present, on the mainland, prevalence rates are higher in urban areas in comparison with rural areas, and vary widely between regions, ranging from $11 \%$ in Iringa to $2.1 \%$ in Dodoma.

The government response ${ }^{2}$ to the epidemic commenced as early as 1985 with the establishment of the National AIDS Control Programme(Ministry of Health 1998), and a number of medium term plans to address the epidemic followed (Ministry of Health 1998). The priorities in the third year plan

\footnotetext{
${ }^{1}$ For a thorough assessment of the spread of the virus across Tanzania, see Illife (2006)

${ }^{2}$ For a detailed view of the resonse to the epidemic in Tanzania, see Garbus, L. (2004). HIVIAIDS in Tanzania. San Fransisco, AIDS Policy Research Center, University of
} 
California 
included targeting commercial sex workers, vulnerable groups, and also poverty reduction strategies, amongst recognition that gender issues, including access to education for girls and an adverse cultural environment for women, were also important issues to address. Following the DHS surveys, which provided greater accuracy on population prevalence rates, the 2008-2012 plan (Ministry of Health and Social Welfare 2007) continued to pursue strategies that acknowledge a broad range of social drivers. With the growing global focus on treatment, the rollout of Antiretroviral Therapy (ART) was slow, with the WHO estimating that there were no patients in on ART (WHO 2002). By 2005, 19,600 individuals were on ART, though this was less than $7 \%$ of those requiring it (WHO 2005). However, supported by large volumes of external funding, ART coverage was estimated to have risen to $69 \%$ by 2012 (The Global Fund 2013), though a significant funding gap remains if Tanzania are to achieve the $90 \%$ treatment coverage by 2020 targets set by UNAIDS in 2014 (UNAIDS 2014).

Tanzania has arguably the most comprehensive nationally representative data available on HIV prevalence, with three HIVIAIDS indicator surveys now completed (as noted above). Following Parkhurst (2010), we include the most recent data from the 2011/12 Tanzanian survey to update his analysis ${ }^{3}$. Below, we conduct a trend analysis over three time periods 2003-4, 2007-8 and 2011-12. An important qualifier to emphasise is that this data is not cohort, or panel, data: it does not track the incidence of HIVIAIDS transmission within a closed group of respondents - instead, it gives three separate 'snapshot' pictures of the prevalence at different intervals. However, it is to be hoped that, with broad population coverage, each snapshot is reasonably representative and can give us some insight into the changing distribution of HIVIAIDS in Tanzania over the last decade.

The measure of wealth in the DHS surveys is based on an 'asset index' incorporating a range of indicators including ownership of certain consumer durables, housing conditions, water access and so on, to meaningfully rank individual respondents' relative wealth in one of five quintiles for the country. This approach tends to correspond poorly with those rankings produced using monetary measures such as income or expenditure and alternative explanations have been put forward for this (Johnston 2013): however, monetary measures can fluctuate particularly dramatically in poorer countries where incomes can be very volatile, or come from a mixture of formal and informal economic activity poorly suited to easy ranking. In these circumstances, asset measures seem likely to give a more reliable picture of a household's long-run welfare levels, and indeed have been shown to perform well in predicting health and educational outcomes (Wall and Johnston 2008): this evidence suggests it is suitable for this area. The data available for the first period excludes Zanzibar; for subsequent periods data is available for Zanzibar but in the interest of comparing like with like, we continue to exclude Zanzibar from the analysis ${ }^{4}$.

There are two major criticisms to address before undertaking this quantitative analysis dealing with highly-aggregated data. The first comes from Bujra (2006), who correctly points out that wealth groups are not a perfect substitute for 'class', and that much qualitative understanding can be obscured by an overly-quantitative, aggregated focus: true, 'wealth quintile' does not adequately capture 'class' - the profoundly social (and sexual) relations upon which the spread of HIVIAIDS is predicated cannot be represented in a headcount which applies these asset-based distinctions and

\footnotetext{
${ }^{3}$ All data is available from www.dhsprogram.com

${ }^{4}$ Incidentally, the inclusion of Zanzibar does not substantially alter the overall impression of the data.
} 
cannot portray interrelations within and between the corresponding groups of people. This detracts from putting forward our results as a complete answer: rather, the 'wealth quintile' comparisons should be situated within, and compared against, qualitative studies which look to understand those interrelations; furthermore, this analysis occupies an important middle ground between (irreplaceable) qualitative and theoretical frameworks, and the inevitable top-level analysis of the highest aggregation - it prevents lazy conclusions that since overall prevalence has declined, this decline must be uniform across the population. There can be no single optimal level of analysis which all studies must follow; far greater understanding can emerge from reading across studies conducted at different levels of analysis.

The second major criticism (Gillespie et al 2007) concerns the inadequacy of the terms 'rich' and 'poor' in low-income countries where almost all are 'the poor'. This criticism, though important, is in large part semantics: even if three or four quintiles of the population come below a given poverty line, this does not imply homogeneity of life quality or prospects among this population majority.

The relational terms 'richer' and 'poorer' still apply. For one example, an economicallyinduced decision to sell unsafe sex once or twice during temporary hardship is not equivalent to the economically-induced decision to sell unsafe sex repeatedly during prolonged hardship, in terms of life quality or risk of infection.

Below we present data on HIV prevalence rates and testing refusal rates. Table 1 shows HIV prevalence, by gender and wealth quintile, across all three time periods; it also shows the chi-square trend test which is shown to be statistically significant in all cases. Figures 1 , 2 and 3 show the prevalence rates graphically, split by gender and wealth quintile, for each time period, with $95 \%$ confidence intervals constructed. Table 2 shows the proportion of survey respondents who declined the HIV blood test also by wealth quintile and gender. It is worth noting that overall coverage of HIV testing was influenced by some potential respondents not being interviewed, and also that the measure for refusal to tests is different in the first survey. 
Table 1: Tabulated HIV prevalence in Tanzania across three time

periods. (All data: DHS/AIS) Figure 1: HIV prevalence in Tanzania,

2003-04. (Data: DHS/AIS.)

Figure 2: HIV prevalence in Tanzania, 2007-08. (Data: DHS/AIS.)

Figure 3: HIV prevalence in Tanzania, 2011-12. (Data: DHS/AIS.)

A number of simple observations regarding the socioeconomic distribution of HIVIAIDS can be made. First, in all cases the chi-square indicates the presence of a trend between wealth quintile and prevalence. It is clear that the overall prevalence is declining; however, there are a number of examples where the prevalence within a wealth quintile rises from one period to the next (for example, among poorer women between 2003-04 and 2007-08). It is also evident that prevalence among women is consistently and significantly greater than that among men, by a far greater factor than the 1:1.2 ratio estimated by the earlier UNAIDS data (Bennel 2004).

The results for the earliest period demonstrate a positive monotonic relationship between wealth and prevalence for both sexes, particularly strong for women: the prevalence of the wealthiest quintile of women being almost four times as great as the poorest. In the second period the relationship between prevalence and relative wealth becomes less straightforward, in line with the hypothesis that, as an epidemic matures, the brunt of new infections passes to poorer individuals as wealthier and better-educated people respond to public health messages: among men, it takes on the U-shaped distribution Parkhurst (2010) finds in a number of other African countries, with the decline more pronounced among the middle and richer quintiles. Among women, the relationship is less clear still: significant decreases in the prevalence for the wealthier and middle wealth quintiles are matched by increases in the two poorest quintiles. However, a wealthy woman is still more than twice as likely to be HIV-positive as one of the poorest: a notable narrowing of the gap from the previous period, but still a stark contrast.

The shifts in the relationship between relative wealth and HIV prevalence are different again between the second and third data periods. Again, the overall prevalence declines - more significantly for men than for women. For women, declines in the poorer and wealthiest groups, accompanied with slight increases for the third and fourth quintiles, combine to restore the monotonic relationship between wealth and HIV prevalence; 
however, the gap between the prevalence rate of the wealthiest and poorest women continues to narrow. Whilst not as strongly 
pronounced as in the earlier distribution, the importance of relative wealth as a risk factor is reiterated, and the hypothesis of maturing epidemics shifting 'downwards' through the class system ${ }^{5}$ is challenged by this finding. The distribution for men in 2011-12 is less clear: a decline in prevalence is observable for each quintile except the middle (which experiences a very slight rise) and the wealthiest quintile retains the greatest prevalence, but there is no straightforward pattern to the wealth-prevalence relationship. This should not imply that relative wealth or poverty has become irrelevant to the HIVIAIDS epidemic - and how could it, when it remains such a clear factor in the female experience of HIVIAIDS? Instead, it again reinforces that our understandings should be nuanced and context-specific, and should consider a number of specific pathways which may seem to negate each other's visibility at this level of analysis.

It is important to reiterate here that 'relative wealth' is not a fixed variable; indeed, both 'common sense' and substantial evidence (Nombo 2007; Hodge 2008) indicate that households affected by HIVIAIDS typically face the double burden of a temporary increase in their necessary expenditure (particularly healthcare and food) and a decrease in their productivity and income (through loss of labour to disease, death, and caring for family members). It is therefore difficult, when considering the increase in prevalence among poorer quintiles and the decrease in prevalence among richer or middle quintiles, to say whether we are primarily seeing a redistribution of disease in accordance with socioeconomic factors, or a redistribution of wealth in accordance with sickness. Mishra et al (2007) attempt to control for this potential endogeneity problem by excluding households where HIV-positive individuals had reported being 'seriously ill' for three or more months of the previous year, and found this adjustment had virtually no effect on the observed wealth association.

Naturally, neither 'wealth' nor 'poverty' can be, in their own right, a direct cause of bloodborne disease: it is thus unsurprising that no firm conclusions can be drawn from this analysis in either direction. However, what does emerge is the existence and relevance of a multifaceted and changing relationship between wealth, poverty and HIVIAIDS, supporting (and supported by) the role of multiple channels and mechanisms in either direction.

\section{[Table 2 - The percentage who refused to provide a blood sample for HIV testing, by wealth quintile, for men and women]}

Of course, 'prevalence' and 'incidence' are not conceptually interchangeable: it is hard to assess from this non-panel data how much we might attribute the consistently higher prevalence of HIVIAIDS among the wealthiest quintile of Tanzanian society to the risk factors relative wealth induces, and how much must be disregarded as simply representing the greater longevity of wealthier people who are HIV-positive, as compared with their poorer compatriots. This emphasises the importance of the testing refusal. As Table 2 shows, testing refusal rates for every period demonstrate that the wealthiest are not more likely to agree to provide a blood sample for a HIV test. The large differences between the 2003/04 data and the other to surveys reflects a change in measurement methodology rather than rapid changes in social attitudes towards testing, though this may have been a factor. In general, women are more likely to agree to a test than men, with

${ }^{5}$ Bloom et al 2001, cited in Bujra 2006; the authors note again that relative wealth is not a perfect proxy for class identity 
men in the wealthiest quintile the most likely to refuse a test in all three surveys. There have been significant changes over time for women in the wealthiest quintile, with the refusal rate dropping from $9.5 \%$ to $5.9 \%$ between the $2007 / 8$ and $2011 / 12$ surveys, though this reduction was not observed for men. Again, as with the prevalence data, the evidence reported here would seem to challenge standard assumptions around the behaviour of the wealthy. This data is also telling as it is not based on self-reported data, rather this is data capturing what people actually do, removing any potential biases concerning the wealthy providing more socially acceptable answers, an issue that plagues self-reported data regarding sexual behaviour (Nnko et al. 2004) .

Above all, there are four key points to summarise from our analysis. Firstly, that the overall decline in national prevalence rates is not consistently reflected across all population subgroups. Secondly, that the dynamic distribution of prevalence is significantly different among women and men. Thirdly, that the shape of the distribution, and the distribution of the overall reduction in prevalence, is shifting through time and the spread may be narrowing, but relative wealth is still clearly of great relevance and it remains deeply inaccurate to characterise HIVIAIDS as a disease of poverty in Africa. Finally, data on testing refusal also poses a major problem for the poverty narrative, with the wealthiest consistently most likely to refuse testing.

\section{Reflections on the evidence}

Amongst the studies that focus on poverty, there are some explanations forwarded to explain the prevalence patterns discussed here. These include the notion that the wealthy are more mobile (Fenton, 2004), and have more chances to engage in extra-marital sexual encounters, that the wealthy have a greater ability to maintain concurrent relationships (Shelton et al 2005), and that due to a westernisation of lifestyles and patterns of urbanisation, they have greater access to multiple partners (Gillespie et al, 2007). However, beyond these suggestions, there is little, if any, research on this issue, emphasising the urgent need for this to be corrected in future studies.

Perhaps unsurprisingly, and reflective of the broader policy environment, the initial response by mainstream economics was also predominantly couched in the poverty narrative. Neoclassical analysis (by no means an extensive oeuvre) has failed to produce a convincing explanation for the distribution of HIV prevalence demonstrated in numerous large-scale studies, including this paper: this failure has severe consequences for effective policymaking. Problematic assumptions pervade rational-agent models (such as those of Philipson and Posner 1995, Oster 2005, Oster 2012), where sex - or unprotected sex - is treated as a freely negotiated contract, fully abstracted from the other social relations that connect both parties to each other and to wider social networks. Decision- making about sex is reduced to a risk analysis of infection: intimacy, pleasure, childbearing and social/familial relations are conspicuous by their absence. This article does not pretend to conduct a comprehensive rebuttal of this work (Christensen 1998 provides an extensive account of the logical and empirical problems in the assumptions underpinning Philipson and Posner's model, which (Gersowitz 2005) corroborates in an African context): suffice to say, it is of no surprise that a framework so narrow and lacking in context is incapable of explaining how wealth persistently correlates with higher prevalence, or why wealthier and better-informed (?) agents appear to disproportionately decline testing. Philipson and Posner's 'shadow price' concept of infection, and Oster's (2012) emphasis on the significant role of non-HIV life expectancy in behavioural responses 
to a local epidemic, both lead problematically to the perception of longevity as a policy option: this renders the problem of HIVIAIDS rather circular, as shorter (non-HIV) life expectancy is seen to lead to high levels of HIV prevalence, whilst high prevalence rates contribute to a decrease in overall life expectancy.

The most convincing explanations that address both the roles of wealth and poverty are rooted in the political economy tradition. Bujra (2006) places the AIDS crisis specifically within the context of class formation: the creation of a wage-labour class, with the attendant migration, social upheaval, and severing of familial and community ties and norms; the emergence of a parasitic 'political class' intent on consolidating their own power; and around these, other class fractions of professionals, of domestic labourers, and of those working in the informal sector, dependent on the functioning of other groups for their own class survival. This creates two particular class-related tendencies which combine to shape 'sexual networking': firstly, such networks reproduce and reinforce class fractions (marriage and procreation); secondly, these networks are used to assert power, typically via extra- or non-marital relations. Thus we can consider the specific mechanisms through which high HIV risk is mediated, in this context of major social transformation: migration, changes to family and community structures, and the broader scope for transactional sexual relations are experienced in varied but class-specific ways.

Bujra (ibid) is scathing about the capacity of indexical accounts to fully capture relational dynamics, and not without reason: quintiles of relative wealth do not properly approximate the power dynamics between and within social classes as identified in the sociological tradition. However, we believe useful insight is also to be found in large-scale quantitative analysis: quantitative studies such as this one do inevitably suffer from some collapsing of analytical categories; we venture it not as an entire foundation of proof, but as one corroborating account. Data alone cannot possibly drive our understanding of social processes, but it can be very instructively discussed alongside relational accounts, and yet there have been very few attempts to combine this particular balancing act. Bujra dismisses indexical findings as so pervasive they are 'taken for granted': we rather find them - and specifically the insistent correlation between high socioeconomic status and high prevalence - to be widely acknowledged and disregarded.

A significant difference between the neoclassical accounts and a political economy approach is the orientation of the former towards a concept of agent 'choice', and of the latter towards agent 'capacity'. Hunter (2002) and Leclerc-Madlala (2003) both write with reference to South Africa, but much of their insights should at least give us pause for thought in the Tanzanian context.

Characterising 'capacity' should not be construed as perpetuating a 'passive victim' discourse, and indeed both authors strongly acknowledge the agency of women in negotiating sex and relationships within a context of normalised transactional sex (Hunter 2002; Leclerc-Madlala 2003). Much has been made of the role of prostitution in transmission of HIV, but frequently in terms of easily- identifiable sex work, loosely correlated with the desperation of poverty, with ignorance about HIV transmission or condom use - an approach which again fails to explain persistently high levels of prevalence among the wealthier members of Tanzanian society. Both authors, however, identify transactional sex as written into the very fabric of social relations: the privileged economic position of men (underwritten by both 'traditional' and capitalist relations), masculine discourses which place high value on men having many sexual partners (likewise with roots or justifications to be found in a wide range of indigenous and Western-imported cultural factors), and the agency of women who 
recognise a commodity value placed on sex and exploit it to meet their material 'needs' or desires. The materiality of sexual relations is no longer perceived as contingent on desperate poverty, and economic considerations enter into the sexual decision-making of relatively wealthy women: the narrative becomes compatible with our data findings.

\section{Further implications}

This can of worms regarding 'aberrant' sexual behaviour as in fact normal, prevalent among the wealthy, and socially sanctioned along multiple cultural lines, is a discourse which implicates a high number of relatively wealthy citizens in 'bad' risky sex may well represent too unsettling an introspection to be entertained seriously. However, failure, in academic or policy circles, to engage with intellectual honesty in discussions about the social relations driving HIV infection is problematic for appropriate research and policy. Following Hunter and Leclerc-Madlala's positioning of toxic masculinities and the commodification of sex in the global context, a similar silence might well be only to be expected from powerful voices in the global North: the notion of normalised transactional sex and an emphatic belief that fashionable clothes and expensive cellphones are 'needs' rather than luxuries may feel rather closer to home than any 'educated' or 'sophisticated' commentator, African or Western, wishes to feel about an epidemic. The possibility of such implicit concerns does not make for an environment hospitable to inquiry about the specific processes leading to persistently high rates of HIV prevalence in spite of education and condom access. In policy, much hinges upon the extent to which the more powerful groups in society (specifically, relatively wealthy men) perceive HIVIAIDS to be a personal threat, and the price they accord that threat as compared with the price of structural change. As Akeroyd (2004) asks, even if female empowerment were to substantially reduce the social risk of HIV infection, how does the loss of male 'power over' necessitated by female empowerment compare to this gain (Akeroyd 2004)? There are powerful beneficiaries of female precarity in the era of HIVIAIDS; agents and institutions for whom the circular relationship between inequality and HIVIAIDS is a rewarding one.

Such a lack of rigorous engagement undermines the capacity of any approach aimed at unpicking and addressing the structural drivers of HIV: if the behaviour of the wealthy becomes difficult to discuss, the 'structural drivers' agenda melts too easily back into the poverty narrative and leaves us with piecemeal policies such as microfinance and conditional cash transfer schemes - approaches which can only comprehend the transmission of HIV within a context of absolute material deprivation, and within a very specific characterisation of gender and economic power. Whilst the majority of women's economic empowerment programmes include microfinance, it is clear that the notion of empowerment has taken on a distinct concrete form, with wealthy women excluded from this conceptualisation. This helps illustrate the way that structural interventions are being captured by individualised and reduced forms of intervention, with microfinance increasingly being labelled as a 'structural' intervention. Further, this is an approach that focuses on protecting poor(er) women from wealthier men, presented on a range of stylised assumptions about gendered roles (O'Laughlin 2008). A 'structural' intervention would address the underlying social structures, processes and relations that shape women's economic dependence on men in general, rather than simply mediating individual impoverished women's economic dependence on men within the existing distribution of (access to) resources. This creeping capture of 'structural' responses illustrates the precarity of Baylies' (2000) optimistic contention that, since the nature of the pandemic so clearly 
implicates structural inequality, it also makes structural change both possible and necessary : there is no failsafe mechanism to ensure that such structural change will occur.

There are further possible 'losers' from a radical rethink of HIVIAID“ policy. Hunsmann (2012) identifies the political economy of an 'AID“ industry' which forms an effective political constituency for no change to existing infection prevention policies: powerful advocates are concerned to maintain their own funding streams, and 'outsider' groups, potentially pursuing approaches which are more long-term or more appropriate to the changed needs of the changing epidemic, can be disparate and powerless (Hunsmann 2012). This pathdependency in policy can create tensions between short-run and long-run approaches. The social costs faced by civil society, of both HIVIAIDS and policies targeted at it, may be greatly mismatched with the costs (and benefits) as perceived by policy-makers; incentives and timescales in politics can conflict with the long-term interests of the society they govern. For Hunsmann, therefore, a structural response to a structural problem is to focus on reshaping the context of policy-making in the hope of this leading to better long-term policies rather than the common efforts of struggling against the incremental nature of policymaking in search of silver bullets. Whilst it may be difficult to follow up this astute identification of the problem of existing approaches, it points to the challenges of re-orienting policy to address the prevalence, behaviour, and testing and counselling habits of wealthy men, in the context in which policy is predominantly being dictated by wealthy men who are likely to divert attention from their own social group, and who have incentives for the perpetuation of the poverty paradigm.

The account we put forward is emphatically not one of determinism; it is also not one that provides an easy solution. Above all, our intention is to draw attention to questions barely asked in research and policy, and even less answered: why does policy seem not to target wealthy women, when they are consistently experiencing the highest rates of prevalence? How might it do so? How closely do our 'structural' interventions fit the structural, social processes underlying HIV transmission? The data on testing refusal again recalls the limitations of rational agent models for policy-makers, because revealed behaviour does not correspond as anticipated by such models: why are wealthy men least willing to know their serostatus? This is an early result we advance with some caution: it is unclear to what extent these findings can be extrapolated to the general population, as there are a range of other factors which may mean that the wealthy do in fact test more, such as access to testing facilities in urban areas or hospital visits in which patients can be routinely tested for HIV (Obermeyer et al. 2013). Nonetheless, this brings into question whether wealthy individuals may be more likely to test because of similar 'rational' behaviours that are reflected in mainstream economic models, or whether this is just a matter of access. Further, this again challenges stylised negative views of uninformed choices made by poor people. Nonetheless, other issues, such as the stigma around testing for HIV, and concerns over what may happen if the test of positive (Matovu et al. 2014), will not just apply to the poor. The data at least suggests that assumptions around testing and wealth should be treated with caution. Further, this has implications for transmission, as wealthy men have higher rates of HIV and are as likely to refuse a test in comparison to the men in the other four wealth quintiles. Indeed, recent data suggests that men are less likely than women to be on A'V's (UNAIDS 2013). More research is needed urgently on this matter to assess whether these testing refusal patterns are reflective of the general population. 


\section{Conclusion}

Our findings therefore contribute to, and reflect, the burgeoning understanding of HIVIAIDS as a dynamic and complex phenomenon (Gupta et al. 2008; Auerbach et al. 2010;

Auerbach et al. 2011), closely related to and strongly influenced by gender and wealth - and more precisely, the specific social relations and structural mechanisms relating these rather abstract concepts to real life in contemporary Tanzania. They reflect the role of both relative wealth and relative poverty, as structural drivers of illness, and as mediators of policy responses. They highlight not only the uneven distribution of HIVIAIDS but also the uneven distribution of the headline rate of decline among different groups of the population; and they remind us that the 'progress' of the epidemic is often non-linear (as in the increase in prevalence for some groups, or the revived monotonic wealth- prevalence relationship among women in 2011-12). Academic and policy responses need to take into account not only the complexity of this nexus, but also its rapid dynamism if they are to offer relevant explanations or solutions. Further, to talk of a 'link' between either, or both, poverty and prevalence or wealth and prevalence, is overly simplistic. This aggregate-level investigation only confirms the relevance of socioeconomic factors to an irreducibly socioeconomic phenomenon. The critical task is to explore the varied channels through which relative wealth and gender shape risk, behaviour, and constraints. The broad brushstrokes of 'gender', 'wealth', 'poverty' and even 'class' provide only the crucial backdrop against which context-specific mechanisms can be construed.

Mainstream economic models are consistently failing to help us understand the epidemic. The 'rational' model of behaviour is contradicted by the evidence around testing refusal, undermining the view that we do not need to be concerned about the wealthy. In fact, these findings indicate that more research, and more attention to wealth, is required: in particular, the evidence illustrates that wealthy women are missing from responses to the epidemic and need re-including.

One of the clearest and repeated inferences from this research is that a significant gap remains between the most aggregated quantitative analysis, which can help indicate the most affected social groups but are limited in their capacity to explain how this risk is embodied, and much closer qualitative analysis which develops nuanced theories of how 'risk groups' and 'risk behaviours' can interact to form 'risk situations' but which can only be very tentatively generalised.

Bujra (2006) is correct to emphasise that 'relative wealth' is an imperfect proxy for class, and to neglect class relations necessarily renders incomplete any analysis of an epidemic driven almost entirely by social-sexual relations and thus by the power dynamics governing these relations. Further work remains to continue developing a gendered class-based analysis which is structural and non- deterministic, and which is compatible with the observed statistical distribution of prevalence as it changes through time. As HIVIAIDS is long-run and permanent (since infection is irreversible), and policy-sensitive, including in indirect or unanticipated ways, the specifics of context need to play a stronger role in modelling: for example, migration and the issues it raises and policies it is affected by (labour market, housing, restructuring of communal and familial relations) cuts across gender and class and is profoundly shaped by both, but is also a factor and pathway in its own right.

A further need is primarily methodological: as Johnston (2013) points out, cohort studies enable more reliable data, addressing the difficulties arising from the distinction between 'incidence' and 'prevalence': cohort studies would enable us to say more confidently whether a relative decrease in a given group's prevalence is due to fewer new infections, higher 
mortality, or an income effect 
whereby households have systematically been reclassified to a different wealth quintile. However, the practicalities involved in such a study are severe, especially at a large scale and with an eye to ensuring broad coverage especially with regard to destitute or highlymobile respondents.

Furthermore, as Johnston also notes, the few existing such studies still yield contradictory results across the same region, so it is also clear that such research is no panacea. However, it could still provide an important yardstick for data such as that used here, where incidence has to be estimated from the more easily measurable prevalence.

\section{References}

Akeroyd, A. (2004). Coercion, constraints and "cultural entrapments": A further look at gendered and occupational factors pertinent to the transmission of HIV in Africa. HIV and AIDS in Africa: Beyond epidemiology. E. Kalipeni, S. Craddock, J. R. Oppong and J. Ghosh. Oxford, Blackwell.

Asamoah-Odei, E., J. M. G. Calleja and J. T. Boerma (2004). "HIV prevalence and trends in sub- Saharan Africa: no decline and large subregional differences." The Lancet 364(9428): 35-40.

Auerbach, J., J. Parkhurst and C. Caceres (2011). "Addressing social drivers of HIVIAIDS for the long- term response: Conceptual and methodological considerations." Global Public Health: 1-17.

Auerbach, J., J. Parkhurst, C. Caceres and K. Keller (2010). Addressing Social Drivers of HIVIAIDS: Some Conceptual, Methodological, and Evidentiary Considerations. Social Drivers Working Group: Working Papers.

Awusabo-Asare, K. and S. K. Annim (2008). "Wealth status and risky sexual behaviour in Ghana and Kenya." Appl Health Econ Health Policy 6(1): 27-39.

Bachmann, M. O. and F. L. R. Booysen (2003). "Health and economic impact of HIVIAIDS on South African households: a cohort study." BMC Public Health 3: 1414.

Baird, S. J., R. S. Garfein, C. T. Mclntosh and B. Özler (2012). "Effect of a cash transfer programme for schooling on prevalence of HIV and herpes simplex type 2 in Malawi: a cluster randomised trial." The Lancet 379(9823): 1320-1329.

Barnighausen, T., V. Hosegood, I. M. Timaeus and M. L. Newell (2007). "The socioeconomic determinants of HIV incidence: evidence from a longitudinal, population-based study in rural South Africa." AIDS 21 Suppl 7: S29-38.

Beegle, K. and D. de Walque (2009). Demographic and Socioeconomic Patterns of HIVIAIDS Prevalence in Africa. The Changing HIVIAIDS Landscape. E. Lule, R. Seifman and A. David. Washington, World Bank: 81-104.

Bennel, P. (2004). HIVIAIDS in Sub Saharan Africa: The Growing Epidemic? ELDIS discussion paper, ELDIS.

Booysen Fle, R. and J. Summerton (2002). "Poverty, risky sexual behaviour, and vulnerability to HIV infection: evidence from South Africa." J Health Popul Nutr 20(4): 285-288.

Bujra, J. (2006). "Class relations: AIDS \& Socioeconomic privilege in Africa." Review of African Political Economy 33: 113-129.

Chao, A., M. Bulterys, F. Musanganire, P. Abimana, P. Nawrocki, E. Taylor, A. Dushimimana, A. Saah and The National University of Rwanda-Johns Hopkins University AIDS Research Team (1994). "Risk Factors Associated with Prevalent HIV-1 Infection among Pregnant Women in Rwanda." Int. J. Epidemiol. 23(2): 371380.

Chaturaka, R. and R. Senaka (2010). "HIV, poverty and women." International Health 2(1):

9-16. Cohen, M. S., Y. Q. Chen, M. McCauley, T. Gamble, M. C. Hosseinipour, N.

Kumarasamy, J. G. Hakim,

J. Kumwenda, B. Grinsztejn, J. H. S. Pilotto, S. V. Godbole, S. Mehendale, S.

Chariyalertsak, B.

R. Santos, K. H. Mayer, I. F. Hoffman, S. H. Eshleman, E. Piwowar-Manning, L. 
Wang, J. Makhema, L. A. Mills, G. de Bruyn, I. Sanne, J. Eron, J. Gallant, D. Havlir, S. Swindells, $H$. 
Ribaudo, V. Elharrar, D. Burns, T. E. Taha, K. Nielsen-Saines, D. Celentano, M. Essex and T. R. Fleming (2011). "Prevention of HIV-1 Infection with Early Antiretroviral Therapy." New England Journal of Medicine 365(6): 493-505.

Dodoo, F. N.-A., E. M. Zulu and A. C. Ezeh (2007). "Urban-rural differences in the socioeconomic deprivation-Sexual behavior link in Kenya." Social Science \& Medicine 64(5): 1019-1031.

Fenton, L. (2004). "Preventing HIVIAIDS through poverty reduction: the only sustainable solution?" Lancet 364(9440): 1186-1187.

Fortson, J. G. (2008). "The Gradient in Sub-Saharan Africa: Socioeconomic Status and HIVIAIDS." Demography 45(2): 303-322.

Fox, M., S. Rosen, W. MacLeod, M. Wasunna, M. Bili, G. Foglia and J. Simon (2004). "The Impact of HIVIAIDS on labour productivity in Kenya." Tropical Medicine and International Health 9(3): 318-324.

Freedman, J. and N. Poku (2005). "The socioeconomic context of Africa's vulnerability to HIVIAIDS." Review of International Studies 31(04): 665-686.

Garbus, L. (2004). HIVIAIDS in Tanzania. San Fransisco, AIDS Policy Research Center, University of California

Gersowitz, M. (2005). "The HIV epidemic in four African countries seen through the Demographic and Health Surveys." Journal of African Economies 14(2): 191-246.

Gillespie, S., S. Kadiyala and R. Greener (2007). "Is poverty or wealth driving HIV transmission?" AIDS 21 Suppl 7: S5-S16.

Gupta, G. R., J. O. Parkhurst, J. A. Ogden, P. Aggleton and A. Mahal (2008). "Structural approaches to HIV prevention." The Lancet 372(9640): 764-775.

Hamoudi, A. and N. Birdsall (2004). "AIDS and the Accumulation and Utilisation of Human Capital in Africa." Journal of African Economies 13(AERC Supplement 1): i96-i136.

Hargreaves, J. R., L. A. Morison, J. Chege, N. Rutenburg, M. Kahindo, H. A. Weiss, R. Hayes and A. Buvé (2002). "Socioeconomic status and risk of HIV infection in an urban population in Kenya." Tropical Medicine \& International Health 7(9): 793802.

Hope, K. R., Sr. (2001). "Africa's HIVIAIDS Crisis in a Development Context." International Relations 15(6): 15-36.

Hunsmann, M. (2012). "Limits to evidence-based health policymaking: Policy hurdles to structural HIV prevention in Tanzania." Social Science \& Medicine 74(10): 14771485.

Hunter, M. (2002). "The Materiality of Everyday Sex: thinking beyond 'prostitution'." African Studies 61(1): 99-120.

Iliffe, J. (2006). The African AIDS Epidemic: A History. Oxford, James Currey. Johnston, D. (2013). Economics and HIV: The Sickness of Economics. Abingdon, Routledge. Kalichman, S. C., L. C. Simbayi, A. Kagee, Y. Toefy, S. Jooste, D. Cain and C. Cherry (2006).

"Associations of poverty, substance use, and HIV transmission risk behaviors in three South African communities." Soc Sci Med 62(7): 1641-1649.

Kalipeni, E. and J. Ghosh (2007). "Concern and practice among men about HIVIAIDS in low socioeconomic income areas of Lilongwe, Malawi." Soc Sci Med 64(5): 1116-1127.

Kim, J. C. and C. H. Watts (2005). Gaining a foothold: tackling poverty, gender inequality, and HIV in Africa.

Kongnyuy, E. J., C. S. Wiysonge, R. E. Mbu, P. Nana and L. Kouam (2006). "Wealth and sexual behaviour among men in Cameroon." BMC International Health and Human Rights 6(11).

Leclerc-Madlala, S. (2003). "Transactional Sex and the Pursuit of Modernity." Social Dynamics 29(2): 213-233.

Lopman, B., J. Lewis, C. Nyamukapa, P. Mushati, S. Chandiwana and S. Gregson (2007). "HIV incidence and poverty in Manicaland, Zimbabwe: is HIV becoming a disease of the poor?" AIDS 21 Suppl 7: S57-66.

Magadi, M. (2013). "The Disproportionate High Risk of HIV Infection Among the Urban 
Poor in Sub- Saharan Africa." AIDS and Behavior 17(5): 1645-1654. 
Masanjala, W. (2007). "The poverty-HIVIAIDS nexus in Africa: a livelihood approach." Soc Sci $\underline{\text { Med }}$ 64(5): 1032-1041.

Matovu, J. K., R. K. Wanyenze, F. Wabwire-Mangen, R. Nakubulwa, R. Sekamwa, A. Masika, J. Todd and D. Serwadda (2014). "Men are always scared to test with their partners ... it is like taking them to the Police": Motivations for and barriers to couples' HIV counselling and testing in Rakai, Uganda: a qualitative study.

Mbirimtengerenji, N. D. (2007). "Is HIVIAIDS epidemic outcome of poverty in sub-saharan Africa?" Croat Med J 48(5): 605-617.

Ministry of Health, Tanzania,, (2003). Health Sector HIVIAIDS Strategy for Tanzania 20032006. Dar Es Salaam.

Ministry of Health and Social Welfare, T. (2007). Health Sector HIV and AIDS Strategic Plan (HSHSP) 2008-2012. Dar Es Salaam.

Ministry of Health, T., , (1998). The Third Medium Term Plan (MTP-III) for Prevention and Control of HIVIAIDS/STD's 1998-2002. Dar Es Salaam, Ministry of Health, Tanzania, National AIDS Control Programme.

Mishra, V., S. B.-V. Assche, R. Greener, M. Vaessen, R. Hong, P. D. Ghys, J. T. Boerma, A. Van Assche,

S. Khan and S. Rutstein (2007). "HIV infection does not disproportionately affect the poorer in sub-Saharan Africa." AIDS 21: S17-S28.

Msisha, W. M., S. H. Kapiga, F. Earls and S. Subramanian (2008). "Socioeconomic status and HIV seroprevalence in Tanzania: a counterintuitive relationship." International Journal of Epidemiology 37(6): 1297-1303.

Mufune, P. (2014). "Poverty and HIVIAIDS in Africa: Specifying the connections." Social Theory \& Health Advanced online 29th October 2014: 1-29.

Nnko, S., J. T. J. T. Boerma, M. Urassa, G. Mwaluko and B. Zaba (2004). "Secretive females or swaggering males?: An assessment of the quality of sexual partnership reporting in rural Tanzania." Social Science \& Medicine 59(2): 299310.

O'Laughlin, B. (2008). Missing Men Again? Gender, AIDS, and migration in southern Africa. Summary of IGS/CCCRW Commemorative Lecture for Audrey Richards.

Obermeyer, C. M., S. Bott, R. Bayer, A. Desclaux, R. Baggaley and a. t. M. S. Group (2013). "HIV testing and care in Burkina Faso, Kenya, Malawi and Uganda: ethics on the ground." BMC International Health and Human Rights 13(1): 6.

Obermeyer, C. M., M. Neuman, A. Hardon, A. Desclaux, R. Wanyenze, O. Ky-Zerbo, P. Cherutich and

I. Namakhoma (2013). "Socio-economic determinants of HIV testing and counselling: a comparative study in four African countries." Tropical Medicine \& International Health 18(9): 1110-1118.

Oster, E. (2005). "Sexually transmitted infections, sexual behavior and the HIVIAIDS epidemic." Quarterly Journal of Economics 120(2): 467-515.

Oster, E. (2007). HIV and Sexual Behaviour Change: Why not Africa? Cambridge, MA, NBER.

Oster, E. (2012). "HIV and Sexual Behaviour Change: Why not Africa?" Journal of Health Economics January 2012(31): 35-49.

Parkhurst, J. (2010). Understanding the correlation between structural factors of wealth and poverty with HIV in Africa: no single correlation, WHO.

Philipson, T. and R. A. Posner (1995). "The Microeconomics of the AIDS Epidemic in Africa." Population and Development Review 21(4): 835-848.

Seeley, J., S. Dercon and T. Barnett (2010). "The effects of HIVIAIDS on rural communities in East Africa: a 20 -year perspective." Tropical Medicine \& International Health 15(3): 329-335.

Shelton, J. D., M. M. Cassell and J. Adetunji (2005). Is poverty or wealth at the root of HIV. Lancet, Lancet. 366: 1057-1058.

Stillwaggon, E. (2002). "HIVIAIDS in Africa: Fertile Terrain." Journal of Development Studies 38(6): 1. TACAIDS (2005). Tanzania HIVIAIDS Indicator Survey 2003-04, Tanzania Commission for AIDS. 
TACAIDS, Zanzibar AIDS Commission (ZAC), National Bureau of Statistics (NBS), Office of the Chief Government Statistician (OCGS) and ICF International 2013 (2013). Tanzania HIVIAIDS and Malaria Indicator Survey 2011-12. Dar Es Salaam, Tanzania, TACAIDS, ZAC, NBS, OCGS, and ICF International.

The Global Fund (2013). Program Scorecard: Tanzania HIVIAIDS, The Global Fund. Thigpen, M. C., P. M. Kebaabetswe, L. A. Paxton, D. K. Smith, C. E. Rose, T. M. Segolodi, F. L. Henderson, S. R. Pathak, F. A. Soud, K. L. Chillag, R. Mutanhaurwa, L. I. Chirwa, M. Kasonde, D. Abebe, E. Buliva, R. J. Gvetadze, S. Johnson, T. Sukalac, V. T. Thomas, C. Hart, J. A. Johnson, C. K. Malotte, C. W. Hendrix and J. T. Brooks (2012). "Antiretroviral Preexposure Prophylaxis for Heterosexual HIV Transmission in Botswana." New England Journal of Medicine 367(5): 423-434.

UNAIDS (2013). Access to Antiretroviral Therapy in Africa: Status Report on ProgressTowards the 2015 Targets. Geneva, UNAIDS.

UNAIDS (2013). Global Report on the Epidemic 2013. Geneva, UNAIDS.

UNAIDS (2014). 90-90-90: An ambitious treatment target to help end the AIDS epidemic. Geneva, UNAIDS.

Wall, M. and D. Johnston (2008). "Counting Heads or Counting Televisions: Can Assetbased Measures of Welfare Assist Policy-makers in Russia?" Journal of Human Development 9(1): 131-147.

Weiser, S. D., K. Leiter, D. R. Bangsberg, L. M. Butler, F. Percy-de Korte, Z. Hlanze, N. Phaladze, V. lacopino and M. Heisler (2007). "Food Insufficiency Is Associated with High-Risk Sexual Behavior among Women in Botswana and Swaziland." PLoS Med 4(10): e260.

Whiteside, A. (2002). "Poverty and HIVIAIDS in Africa." Third World Quarterly 23(2): 313-332.

WHO (2002). Coverage of selected health services for HIVIAIDS prevention and care in less developed countries in 2001. Geneva, WHO.

WHO (2005). Summary of Country Profile for HIVIAIDS Treatment Scale-up. Geneva, WHO.

Wilson, D. and D. T. Halperin (2008). "'Know your epidemic, know your response": a useful approach, if we get it right." The Lancet 372(9637): 423-426.

Wojcicki, J. M. (2005). "Socioeconomic Status as a Risk Factor for HIV Infection in Women in East, Central and Southern Africa: A Systematic Review." Journal of Biosocial Science 37(01): 1-36.

World Bank (1999). Confronting AIDS; Public Priorities in a Global Epidemic. New York and Washington, Oxford University Press and World Bank. 